\title{
Más allá de la convención de México y los principios de la Haya: ¿Qué sigue para las américas?*
}

José Antonio Moreno Rodríguez ${ }^{* *}$

\section{DESARROLLOS EN LOS SIGLOS XIX Y XX}

Movimientos nacionalistas en Europa y las Américas frenaron la idea de un derecho universal, alejándose del ius commune y la lex mercatoria que se habían consolidado en tiempos medievales. A lo largo de las jurisdicciones con tradición civilista, las naciones-estados sancionaron códigos civiles y comerciales, mientras que países de tradición anglosajona desarrollaron su derecho autóctono en base a precedentes jurisprudenciales. Esto, a su vez, ha dado un peculiar impulso al derecho internacional privado, como una disciplina destinada a resolver el rompecabezas del "conflicto de leyes" -o conflictualismo-, en tiempos en que las soluciones nacionales que buscaban abordar el problema resultaron alarmantemente contradictorias". "Lugar de ejecución", "lugar de cumplimiento"y otras fórmulas fueron, por ejemplo, propuestas para resolver temas referentes al derecho aplicable a un contrato internacional.

El jurista alemán Savigny, ha sido muy influyente hacia mediados del siglo XIX, con su idea de unificar estas fórmulas en un tratado internacional que vinculara a todas las naciones que lo ratificaran ${ }^{2}$. Esta propuesta condujo a

\footnotetext{
* Traducción al español a cargo de José Antonio Moreno Bendlin bajo la supervisión de Lucía Cazal, de la ponencia preparada para el encuentro "Towards a Global Framework for International Commercial Transactions" (Lucerna, Suiza, 9 de setiembre de 2016), organizado por la Universidad de Lucerna y la Oficina Permanente de la Conferencia de La Haya de Derecho Internacional Privado. ** LL.M, Harvard. Miembro del Comité Jurídico Interamericano de la Organización de Estados Americanos. Miembro del Grupo de Trabajo de la Conferencia de La Haya de Derecho Internacional Privado en materia de contratación internacional. Autor de la ley paraguaya de contratos internacionales. Presidente de la Asociación Americana de Derecho Internacional Privado. Miembro del Consejo de Gobierno de UNIDROIT. Miembro de la Corte de Arbitraje de la Cámara de Comercio Internacional. Profesor de grado y posgrado en diversas universidades de Paraguay y el extranjero, entre ellas Heidelberg y Paris II. www.jmoreno.info
} 
la inauguración de la Conferencia de la Haya de Derecho Internacional Privado en 1893, bajo el liderazgo del jurista holandés Asser³. Sin embargo, como se verá más abajo, más de cien años pasarían antes de que la Conferencia de la Haya -convertida en cuerpo permanente en 1955- pasara a ocuparse del derecho aplicable en contratos internacionales.

Mientras tanto, las Américas tomaron la delantera. Uno de los Tratados de Montevideo de 1889, firmados en esa misma ciudad (el de Derecho Civil Internacional), específicamente aborda la cuestión de elección del derecho aplicable, aunque adoptando soluciones altamente controversiales en cuanto a la ausencia de elección, y guardando silencio en relación a la autonomía de las partes $^{4}$. Estos antiguos Tratados de Montevideo siguen siendo aplicables entre Argentina, Bolivia, Colombia, Paraguay, Perú y Uruguay.

En 1940, nuevos tratados fueron firmados en Montevideo (ratificados solo por Argentina, Paraguay y Uruguay) que reafirmaron las antiguas soluciones con respecto a la ausencia de elección del derecho aplicable y una regla general- con excepciones- de la aplicabilidad del derecho del lugar de cumplimiento. Además, estos siguientes tratados dispusieron que cada estado debería determinar por su cuenta la aceptación -o no- del principio de autonomía de las partes, una cuestión que, ante la ausencia de provisiones claras a ese efecto en legislaciones domésticas, se tornó muy controvertida en Brasil ${ }^{5}$, Paraguay ${ }^{6}$ y Uruguay 7 .

Muchos otros estados en el continente, como Brasil, Chile y Venezuela, no han incorporado los Tratados de Montevideo. En cambio, han ratificado el llamado "Código Bustamante"de 1928, que fue adoptado como resultado de la sexta conferencia Pan- americana desarrollada en La Habana, Cuba, en 1928. Este Código, que regula varias cuestiones de derecho internacional privado, entre ellas el derecho aplicable a la contratación internacional, ofrece una solución distinta ante la ausencia de elección del derecho aplicable, a saber, la aplicabilidad del derecho del lugar de ejecución. Así también, el instrumento ha levantado muchas interrogantes con relación a si consagra o no la autonomía de las partes ${ }^{8}$.

Para mediados del siglo XX, existía un sentimiento generalizado de que los documentos mencionados previamente, adoptados en las Américas, resultaban insatisfactorios, primero, debido a las soluciones cuestionables que proponían, y segundo, debido a las divergencias entre los mismos. Para empeorar la situación, algunos estados Americanos -notablemente, aquellos de tradición anglosajonano habían ratificado ninguno de estos instrumentos.

El establecimiento de la Organización de Estados Americanos (OEA) en 1948 generó fuertes esperanzas de que la situación fuese finalmente resuelta. Tras cuidadosas evaluaciones, la OEA decidió en contra de la idea de elaborar un código general como el de Bustamante, y, en su lugar, optó por trabajar hacia la codificación gradual de temas particulares en el ámbito de derecho internacional privado $^{9}$. Esta idea empezó a convertirse en una realidad en 1975 con la primera Conferencia Especializada Inter-Americana sobre Derecho Internacional Privado 
(CIDIP), que fue convocada para adoptar ciertos instrumentos en temas como arbitraje y otros de derecho comercial y procesal internacional. Hasta la fecha, siete CIDIPs han sido llevadas a cabo, las que han resultado en la adopción de 26 instrumentos internacionales (incluyendo convenciones, protocolos, documentos uniformes y leyes modelo $)^{10}$.

No fue hasta la CIDIP V, llevada a cabo en la Ciudad de México en el 1994, que se abordó la cuestión de la elección del derecho aplicable en los contratos internacionales. El instrumento resultante fue la Convención Interamericana sobre el Derecho Aplicable a los Contratos Internacionales, conocida comúnmente como la "Convención de México". Este documento claramente reconoce la autonomía de las partes (Artículo 7) y, en ausencia de elección, estipula el uso de la "conexión más cercana" (Artículo 8), la cual puede llevar tanto a la aplicación del derecho nacional o del derecho no estatal. (i.e., principios del derecho comercial internacional, lex mercatoria, etc. $)^{11}$ En todo caso -haya habido o no elección- la Convención de México dispone que el derecho no estatal debe ser tomado en consideración si la justicia o la equidad así lo requieren, lo que está expresado en otras palabras en el instrumento ${ }^{12}$.

Sin embargo, la Convención de México ha sido ratificada solo por dos estados: México y Venezuela.

El instrumento Europeo comparable con relación a la elección de derecho aplicable, conocido como el Convenio de Roma de $1980^{13}$, ha gozado de un destino distinto. Este documento fue adoptado por varios estados Europeos y, en 2008, fue convertido en Reglamento Comunitario 593 de 2008 (llamado "Roma I"), lo que lo hizo aplicable -inicialmente con algunas excepciones- a toda la Unión Europea. El Convenio de Roma -así como la Convención de México- resuelve la cuestión en favor de la autonomía de las partes. Lamentablemente, incluye soluciones criticadas que favorecen el lugar de la prestación característica ante la ausencia de elección ${ }^{14}$, y además descarta la aplicabilidad del derecho no estatal ${ }^{15}$.

El Convenio de Roma se tornó relevante no solo por su adopción por el bloque Europeo, sino también debido a su influencia en el proyecto de elaboración de la Convención de México en las Américas y, más recientemente, en la preparación de un instrumento que aborda la cuestión a una escala global.

\section{HACIA SOLUCIONES UNIVERSALES: LOS PRINCIPIOS DE LA HAYA}

El éxito de la Convención de Roma llevó a la Conferencia de la Haya de Derecho Internacional Privado a encarar estudios de viabilidad a inicios de la década de 1980 sobre la posibilidad de adoptar un instrumento similar a escala global. Este emprendimiento fue descartado tras haber considerado las dificultades de obtener una ratificación masiva del instrumento propuesto, cuya falta haría del 
proyecto un fracaso ${ }^{16}$. Sin embargo, la materia fue revisitada en años recientes, y los estudios de viabilidad que se llevaron a cabo entre el 2005 y 2009 indicaron que tal vez un tipo de instrumento diferente podría resultar exitoso y efectivo ${ }^{17}$.

Con este propósito, un Grupo de Trabajo fue formado en el año 2010 ${ }^{18}$, el cual avanzó en la idea de -en vez de pretender la adopción de un tratado o instrumento de hard law- elaborar un instrumento de soft law, inspirado en cuanto a la técnica de redacción, en los muy elogiados Principios UNIDROIT sobre los Contratos Comerciales Internacionales. Los Principios UNIDROIT desarrollan cuestiones de derecho sustantivo mientras que los Principios de la Haya -como son comúnmente referidos- se limitan a tratar cuestiones de elección de derecho aplicable, específicamente en relación a la autonomía de las partes. La cuestión de la ausencia de elección no fue abordada, debido a que ello hubiese hecho del proyecto uno demasiado ambicioso, y quizás también porque tendría poco sentido regular el tema en un instrumento de soft law ${ }^{19}$. Dado que los Principios de la Haya -así como los Principios UNIDROIT- no están destinados a ser adoptados formalmente por estados, su aplicabilidad normalmente será el resultado de haber sido seleccionados por las partes como derecho aplicable, en ejercicio de la autonomía de la voluntad.

Sin lugar a dudas, también se prevén otras formas de aplicación de los Principios de la Haya, notablemente como un modelo para legisladores y como una herramienta de interpretación para jueces y árbitros, lo que podría justificar su futuro mejoramiento. Sin embargo, como ya fue manifestado, la versión actual de los Principios -adoptada en primer lugar por una sesión diplomática de la Comisión Especial en el $2012^{20}$ y finalmente por una resolución del Consejo en el $2014^{21}$ - solo desarrolla cuestiones relacionadas a las partes y limitaciones a este respecto, prescriptas por el orden público.

Los Principios de la Haya fueron bien recibidos por destacados estudiosos del derecho internacional privado, pero también han sido objeto de cuestionamientos, particularmente en cuanto a su aceptación del derecho no estatal $^{22}$. A pesar de que el nuevo instrumento quizás no ha sido aún sujeto de ningún precedente jurisprudencial, ya ha influenciado de manera notoria la promulgación de legislación en el Paraguay (Ley 5393 de 2015²3) así como una propuesta de reforma al derecho australiano en la materia ${ }^{24} \mathrm{El}$ ejemplo paraguayo volverá a ser mencionado más adelante.

\section{3. ¿Y AHORA QUÉ EN LAS AMÉRICAS?}

Aunque los Principios UNIDROIT hayan influenciado a los Principios de la Haya en cuanto a su técnica de elaboración, éstos fueron sumamente influenciados en cuanto a su contenido, por las soluciones propuestas tanto en el Convenio de Roma como en la Convención de México. 
Más de veinte años han pasado desde la adopción de la Convención de México y, por supuesto, los Principios de la Haya también incorporaron desarrollos subsiguientes que allanaron el camino para encontrar soluciones a ciertas cuestiones nuevas, como, por ejemplo, la separabilidad de la cláusula de elección de derecho aplicable del contrato principal. Más aún, este instrumento de soft law ha incorporado mejores soluciones a ciertas cuestiones que, en la elaboración del instrumento interamericano, han sido objeto de soluciones de compromiso debido a la complejidad de las negociaciones cuando se pretende la adopción de un tratado. Esta situación se dio, particularmente, con relación al derecho no estatal.

Ahora, ¿qué sigue para las Américas? ¿Insistirá la región en la ratificación de la Convención de México? ¿Debería ser modificada la Convención, tomando en consideración los nuevos desarrollos? ¿Quizás se debería preparar una ley modelo? La última pregunta ha ganado particular impulso tras la promulgación de la nueva ley paraguaya sobre el derecho aplicable a los contratos internacionales.

Recientemente, el Comité Jurídico Interamericano de la OEA analizó todas estas alternativas tras haber circulado un cuestionario entre los estados miembros de la organización y especialistas reconocidos del derecho internacional privado. Las respuestas reflejan la percepción de que, evidentemente, los Principios de la Haya han llegado más lejos que la Convención de México y que sus disposiciones podrían servir para enmendar el documento interamericano ${ }^{25}$.

Sin embargo, considerando que la Convención de México, elaborada en el año 1994, ha recibido solamente dos ratificaciones, la verdadera pregunta es si un proceso para llegar a un convenio nuevo y revisado valdría el esfuerzo. Una posible respuesta es que dicho documento mejorado podría ser mejor recibido por la comunidad jurídica dentro de las Américas, y además que ello daría la oportunidad de corregir la traducción al inglés del instrumento original, la cual fue criticada por juristas angloparlantes.

Pero el proceso de negociación y aprobación de una convención es muy complicado y costoso, mientras que otros tipos de instrumentos -como las leyes modelo y guías legislativas- han demostrado ser medios efectivos para armonizar soluciones de derecho internacional privado. En última instancia, sería mucho más efectivo para los estados latinoamericanos el adoptar leyes nacionales acorde con las prácticas respaldadas por la OEA y por la Conferencia de la Haya, aplicables a todas las transacciones internacionales, en vez de promover la adopción de tratados como la Convención de México y su eventual modificación, lo que afectaría solamente a las partes contratantes de los estados que la hayan ratificado.

Entonces, una ley modelo o una guía legislativa podrían ser una buena idea. Pero, ¿Por qué simplemente una guía "legislativa"? ¿Por qué no una guía que sirva también para jueces, árbitros, partes contratantes y académicos? En respuesta, 
el Comité Jurídico Interamericano ha encargado a tres de sus miembros la elaboración de una guía sobre el derecho aplicable a los contratos internacionales, con el apoyo del Departamento de Derecho Internacional de la OEA ${ }^{26}$. Esta guía será luego sujeta a aprobación formal por el Comité en su totalidad.

La propuesta combina lo mejor de los dos mundos: En primer lugar, esta guía servirá como un instrumento educacional. Esto no es algo menor, considerando que una de las razones por la cual la Convención de México ha encontrado una fuerte resistencia fue por la falta de información con relación a su contenido e implicaciones. Una guía podría superar este obstáculo.

En segundo lugar, la guía será una herramienta disponible para legisladores que tendrá en cuenta desarrollos recientes consagrados en los Principios de la Haya y que también cubrirá materias no abordadas expresamente en los mismos, pero que fueron reguladas en la Convención de México, específicamente con respecto a la ausencia de elección de derecho.

En tercer lugar, la guía puede aprovecharse del hecho de que UNIDROIT, UNCITRAL y la Conferencia de la Haya, están previendo que el documento explique la interacción entre los Principios de UNIDROIT, la Convención de Viena sobre la Compraventa y los Principios de la Haya. Referencia sobre esta interacción se puede hacer, en cambio, con respecto al documento de la OEA.

Finalmente, la guía puede ser una poderosa herramienta interpretativa en la mano de jueces, árbitros y partes, considerando las incertidumbres alarmantes que todavía persisten en la materia de contrataciones internacionales. El instrumento expondrá soluciones basadas en el sentido común y altamente debatidas, que se encuentran tanto en los Principios de la Haya como en la Convención de México, explicando sus complejidades.

\section{CONCLUSIÓN}

Se puede ver la luz al final del túnel. El nacimiento de la Convención de México generó enormes expectativas que no fueron alcanzadas por los acontecimientos que le siguieron, en particular, por el número escaso de ratificaciones, a pesar de los elogios que le han sido conferidos en círculos internacionales de académicos líderes en derecho internacional privado.

Actualmente, el fénix podría estar resurgiendo de entre sus cenizas, como fue evidenciado por dos acontecimientos recientes claves: la adopción de los Principios de la Haya y la promulgación de la nueva ley Paraguaya sobre contratos internacionales. Esta última sirve como modelo de cómo los Principios y la Convención de México pueden interactuar espléndidamente.

Impulsada por estos acontecimientos, la OEA está preparando una Guía para las Américas sobre la Ley Aplicable a Contratos Internacionales, lo que debería coadyuvar a que la Convención de México sea incorporada a los derechos 
nacionales ya sea a través de la ratificación estatal o ya sea por la adopción de sus soluciones en las legislaciones de derecho internacional privado de los estados -en cuyo caso sería deseable incorporar además, los avances acogidos por los Principios de la Haya, como fue hecho en el Paraguay-.

Algunos caminos llevan más allá de Roma y algunos, aún más allá de México. La Conferencia de la Haya -y en las Américas, la OEA- juegan un papel clave en este llamado.

\section{NOTAS}

1 solo desde el siglo XIX (G. Kegel, International Enciclopedia of Comparative Law, Chapter 3, Fundamental Approaches, J. C. B. Mohr (P. Siebeck)/ Tübingen/ and Martinus Nijhoff Publishers/ Dordrecht/ Boston/ Lancaster, 1986, p. 5).

2 M. F. C. de Savigny, Sistema de Derecho Romano Actual, Tomo Sexto, Segunda Edición, Centro Editorial de Góngora, Madrid, p. 137.

3 En 1881 el jurista y político italiano Mancini intentó llevar adelante una conferencia de derecho internacional privado, que no se realizó. La iniciativa fue retomada por el flamenco Asser, bajo cuya influencia el gobierno de su país invitó en 1892 a principales Estados europeos a una conferencia para acordar codificación internacional de conflictos de leyes (M. WoLfF, Derecho Internacional Privado, Traducción española de la segunda edición inglesa por Antonio Marín López, Barcelona, Editorial Bosch, 1958, p. 44). Tal constituye la génesis de la Conferencia de Derecho Internacional Privado.

4 Sobre las críticas, ver, por ejemplo, en D. Hargain/ G. Mihali, Régimen Jurídico de la Contratación Mercantil Internacional en el MERCOSUR, Montevideo/ Buenos Aires, Julio César Faira Editor, 1993, p. 31, p. 39. Sobre el problema de la autonomía de la voluntad en los Tratados de 1889, ver en: R. Santos Belandro, El Derecho Aplicable a los Contratos Internacionales, 2a Ed., Montevideo, Editorial Fundación de Cultura Universitaria, 1998, pp. 55-56.

5 N. DE ARaújo, Contratos Internacionais, 2a Ed., Río de Janeiro, Libraría e Editora Renovar Ltda., 2000, pp. 320-324.

6 Ver mi artículo sobre la "Autonomía de la Voluntad en el Derecho Internacional Privado Paraguayo", en el Libro Homenaje a Tatiana Maekelt, CEDEP, Asunción, 2010, pp. 409 y siguientes.

7 Puede verse un amplio recuento en C. Fresnedo de Aguirre, La Autonomía de la Voluntad en la Contratación Internacional, Montevideo, FCU, 1991. Actualmente, existe un giro importante en este tema, analizado en perspectiva más amplia en el siguiente excelente trabajo: D. OperTTi BADÁN, "El Derecho Internacional Privado en tiempos de globalización", en Revista Uruguaya de Derecho Internacional Privado, Año VI, № 6, Montevideo, Editorial Fundación de Cultura Universitaria, 2005

8 Queda abierto hasta hoy el debate a este respecto, aunque no caben dudas de que en el Código Bustamante sí se admite la autonomía para designar expresa o tácitamente al juez competente para dirimir controversias, en tanto al menos uno de los litigantes tenga nacionalidad o domicilio en el país y en cuanto no exista un "derecho local contrario" (D. P. Fernández Arroyo, Acerca de la Necesidad y las Posibilidades de una Convención Interamericana sobre Competencia Judicial en Casos de Derecho Internacional Privado, pp. 120-121).

9 Ver, por ejemplo, en: J-M. ARRIGHI, "El proceso actual de elaboración de normas Interamericanas", en Jornadas de Derecho Internacional, Córdoba, Argentina, organizadas por la Universidad Nacional de Córdoba y la Secretaría General de la Organización de los Estados Americanos, Subsecretaría de Asuntos Jurídicos, Washington, D.C., 2001.; E. Villalta, "El Derecho Internacional Privado en el 
Continente Americano", en Los servicios en el Derecho Internacional Privado, ASADIP y Programa de Pos-Graduacao em Direito da Universidade Federal de Rio Grande do Sul, Porto Alegre, Brasil, 2014, pp. 23 y siguientes.

10 Ver en: http://www.oas.org/dil/esp/derecho_internacional_privado_conferencias.htm (último acceso: 13 de septiembre de 2016).

11 Así lo entendió Juenger, delegado de los Estados Unidos en las deliberaciones conducentes a la Convención de México. F.K. Juenger, "The Lex Mercatoria and Private International Law", en 60 Louisiana Law Review, 2000, pp. 1133-1148. La relevancia de esta opinión la resalta Siqueiros, redactor del proyecto de dicho instrumento, pues Juenger fue quien propuso la fórmula recogida en este artículo luego de un arduo debate y a modo de compromiso. J.L. Siqueiros, "Los Principios de Unidroit y la Convención Interamericana sobre el Derecho Aplicable a los Contratos Internacionales", en Contratación Internacional, Comentarios a los Principios sobre los Contratos Comerciales Internacionales del UNIDROIT, México, Universidad Nacional Autónoma deMéxico, Universidad Panamericana, 1998, p. 223. Con respecto a una norma similar incluida en la ley venezolana de derecho internacional privado, la Suprema Corte de Venezuela ha sentado que la fórmula de la conexión más cercana lleva a la lex mercatoria, que se encuentra conformada por costumbres comerciales y prácticas. Banque Artesia Nederland, N.V. vs Corp Banca, Banco Universal CA (Exp. 2014-000257), de 2014 (ver en www.unilex.info).

12 El Artículo 10 de la Convención de México dispone: "Además de lo dispuesto en los artículos anteriores, se aplicarán, cuando corresponda, las normas, los usos de comercio y los principios de la contratación preponderantes en el derecho comparado, con la finalidad de realizar las exigencias impuestas por la equidad en el caso concreto".

13 Su denominación es “Convenio Sobre la Ley Aplicable a las Obligaciones Contractuales”.

14 Ver G.A. Bermann, "Rome I: A Comparative View”, en F. Ferrari / S. Leible (eds.), Rome I Regulation, The Law Applicable to Contractual Obligations in Europe, München, Sellier, 2009, p. 350.

15 Ver críticas a esto en: M. J. Bonell, "El reglamento CE 593/2008 sobre la ley aplicable a las obligaciones contractuales ("Roma I") - Es decir, una ocasión perdida", en Cómo se Codifica hoy el Derecho Comercial Internacional. J. BAsedow/ D.P. Fernández Arroyo/ J.A. Moreno Rodríguez (eds..), CEDEP y La Ley Paraguaya, 2010.

16 Ver en: M. Pertegás/ I. Radic, "Elección de la ley aplicable a los contratos del comercio internacional. ¿Principios de La Haya?”, en Cómo se Codifica hoy el Derecho Comercial Internacional, J. BASedow/ D.P. Fernández Arroyo/ J.A. Moreno Rodríguez (eds.), CEDEP y La Ley Paraguaya, 2010, p. 341.

17 Puede accederse a los trabajos preparatorios a través del sitio www.hcch.net.

18 N. B. Cohen (Estados Unidos); The Hon. Justice Clyde Croft (Australia); S. E. Darankoum (Canada); A. Dickinson, (Australia); A. S. El Kosheri (Egipto); B. Fauvarque-Cosson (Francia); L. G. E. Souza Jr. (Brasil); F. J. Garcimartín Alférez (España); D. Girsberger (Suiza); Y. Guo (China); M. E. Koppenol-Laforce (Países Bajos); D. Martiny (Alemania); C. McLachlan (Nueva Zelanda); J. A. Moreno Rodríguez (Paraguay); J. L. Neels (Sudáfrica); Y. Nishitani (Alemania); R. F. Oppong (Reino Unido); G. SAumier (Canadá) e I. Zykin (Rusia). Como observadores se designó a los siguientes miembros: M. J. Bonell (UNIDROIT); F. BorTolotTI (Cámara de Comercio Internacional); T. Lemay (UNCITRAL); F. Mazza (Corte de Arbitraje de la Cámara de Comercio Internacional); K. Reichert (International Bar Association) y P. Werner (International Swaps and Derivatives Association). Más adelante se sumaron al Grupo de Trabajo T. Kadner Graziano (Suiza) y S. Symeonides (Chipre), este último de dilatada trayectoria en el derecho norteamericano.

19 Esto fue discutido ampliamente en las deliberaciones del Grupo de Trabajo en La Haya, de las que fui parte. 
20 http://www.hcch.net/upload/wop/contracts_rpt2012e.pdf (último acceso: 13 de septiembre de 2016)

21 http://www.hcch.net/index_en.php?act=conventions.text\&cid=135 (último acceso: 13 de septiembre de 2016)

22 Se ha dicho que la admisión del derecho no estatal se encontró con los resentimientos de una coalición de "hombres del ayer". S.C. Symeonides, Codifying Choice of Law Around the World, An International Comparative Analysis, Oxford University Press, New York, 2014, p. 143. Algunos critican la admisión en sí del derecho no estatal en el instrumento (R. Michaels, Non-State Law in the Hague Principles on Choice of Law in International Contracts, 2014, http://papers.ssrn.com/ sol3/papers.cfm?abstract_id=2386186, V a y b, y VI), en tanto que otros cuestionan el cambio introducido por la sesión de la Comisión Especial en la Haya de 2012 que alteró la propuesta del Grupo de Trabajo (A. Dickinson, "A principled approach to choice of law in contract", 2 Journal of International Banking and Financial Law, 2013, p. 152).

23 Sobre esta ley, de mi autoría, he escrito en algunas publicaciones, y la más reciente es: J. A. Moreno Rodríguez, "The new Paraguayan Law on international contracts: back to the past?", en Eppur si muove: The age of Uniform Law - Festschrift for Michael Joachim Bonell, to celebrate his 70th birthday, Unidroit (ed.), 2016.

24 Esto fue relatado por Brooke Marshall, en el congreso "Towards a Global Framework for International Commercial Transactions", referido en la nota 1.

25 La Convención Interamericana sobre Derecho Aplicable a Contratos Internacionales y el Avance de sus Principios en las Américas, Documento preparado por el Departamento de Derecho Internacional de la Secretaría de Asuntos Jurídicos de la Organización de los Estados Americanos, accesible en: www.asadip.org/v2/?p=5535 (último acceso: 13 de setiembre de 2016).

26 Los miembros del Comité encargados del tema son Elizabeth Villalta, Gélin Imanes Collot y José A. Moreno Rodríguez. Está previsto que un primer reporte sea entregado en la sesión del Comité de octubre de 2016 en Río de Janeiro (http://www.oas.org/en/sla/iajc/agenda.asp - último acceso, 13 de septiembre de 2016). 


\title{
ALÉM DA CONVENÇÃO DO MÉXICO E DOS PRINCÍPIOS DA HAIA: O QUE VEM A SEGUIR PARA AS AMÉRICAS?
}

\begin{abstract}
Resumo
Como um infeliz legado de eventos ocorridos durante o século XIX, a problemática da escolha da lei aplicável aos contratos internacionais tornou-se caótica, caracterizada por soluções conflitantes em todo o mundo. A Europa tem estado na vanguarda ao abordar eficazmente o problema e tentar resolvê-lo - atualmente através de um regulamento que está em vigor na União Europeia. As Américas tiveram menos sorte, com a circunstância agravante de que na região há uma forte divisão entre o direito civil e a lei anglo-saxônica, o que aumenta a complexidade do assunto. Este artigo expõe os esforços realizados nas Américas com a intenção de resolver o problema e descreve os desenvolvimentos recentes que oferecem um cenário promissor para a região no futuro próximo.
\end{abstract}

Palavras-chave: Contratos internacionais. Direito internacional privado. Solução de conflitos. Tratados internacionais. 


\section{Resumen}

Como desafortunado legado de acontecimientos ocurridos durante el siglo XIX, la problemática de la elección del derecho aplicable a los contratos internacionales se volvió caótica, caracterizada por soluciones conflictivas alrededor del mundo. Europa ha estado a la vanguardia en abordar eficazmente el problema y en intentar resolverlo - actualmente a través de una regulación que se encuentra en vigor en la Unión Europea. Las Américas han tenido menos fortuna, con el agravante de que en la región existe una fuerte división entre el derecho civil y el derecho anglosajón, lo que agrega complejidad a la materia. Este artículo expone los esfuerzos llevados a cabo en las Américas con la intención de resolver el problema y describe los desarrollos recientes que ofrecen un escenario prometedor para la región en un futuro cercano.

Palabras-clave: Contratos internacionales. Derecho internacional privado. Solución de conflictos. Tratados internacionales. 


\title{
BEYOND THE CONVENTION OF MEXICO AND THE PRINCIPLES OF THE HAGUE: WHAT FOLLOWS FOR THE AMERICAS?
}

\begin{abstract}
As an unfortunate legacy of events occurred during the nineteenth century, the problem of choosing the law applicable to international contracts became chaotic, characterized by conflicting solutions around the world. Europe has been at the forefront of effectively addressing the problem and trying to solve it - currently through a regulation that is in force in the European Union. The Americas were less fortunate, with the aggravating circumstance that there is a strong division between civil law and Anglo-Saxon law in the region, which increases the complexity of the subject. This article outlines the efforts made in the Americas to address the problem and describes the recent developments that provide a promising scenario for the region in the near future.
\end{abstract}

Keywords: International contracts. International private law. Conflict resolution. International treaties. 